Ciencia y Salud, Vol. IV, No. 1, enero-abril, 2020 •ISSN (impreso): 2613-8816 • ISSN (en línea): 2613-8824

DOI: https://doi.org/10.22206/cysa.2020.v4i1.pp57-70

\title{
INCIDENCIA DE ENFERMEDAD DEPRESIVA EN ADULTOS DE LA TERCERA EDAD, EN ARROYO HONDO, PERAVIA, REPÚBLICA DOMINICANA, DURANTE EL PERÍODO NOVIEMBRE-DICIEMBRE 2018
}

\section{Incidence of depressive disease in elderlyadults, in Arroyo Hondo, peravia, Dominican Republic, during the period november - december 2018}

\section{Jean C. Castillo ${ }^{a}$, Gladisder Díaz Vásquez ${ }^{b}$, Jaylene Reyes Montillac, Secundino Palacios ${ }^{\mathrm{d}}$, Ysabel Díaz Castro ${ }^{\mathrm{e}}$, Emilton López ${ }^{\mathrm{f}}$ y Leopoldo Reyes Nin ${ }^{\mathrm{g}}$}

Recibido: 22 de septiembre, 2019 • Aprobado: 24 noviembre, 2019

Cómo citar: Castillo J, Díaz Vásquez G, Reyes Montilla J, Palacios S, Díaz Castro Y, López E, Reyes Nin L. Incidencia de enfermedad depresiva en adultos de la tercera edad, en Arroyo Hondo, Peravia, República Dominicana, durante el período noviembre-diciembre 2018. cysa [Internet]. 3 de marzo de 2020 [citado 4 de marzo de 2020];4(1):57-0. Disponible en: https://revistas.intec.edu.do/index. $\mathrm{php} / \mathrm{cisa} /$ article/view/1665

Resumen

Introducción: la enfermedad depresiva en las personas mayores de 65 ańos constituye un problema generalizado y desatendido. La etiología es multifactorial; puede ser desencadenada por diversos fenómenos y asociado a patologías en las que, a menudo, se minimizan los síntomas afectivos. En República Dominicana se estima que más de la mitad de los envejecientes padece este trastorno.

Objetivo: estimar la incidencia de enfermedad depresiva en adultos de la tercera edad en Arroyo Hondo, Peravia, República Dominicana, durante el período noviembre-diciembre 2018.

Metodología: estudio prospectivo, descriptivo y de corte transversal. Se entrevistaron 201 personas mayores de 65 años y se les aplicó la Escala de Depresión Geriátrica (GDS-15).

\begin{abstract}
Introduction: Depressive illness in the elderly constitutes a generalized and unattended issue. The etiology is multifactorial; it can be triggered by diverse phenomena and associated with pathologies in which, often, affective symptoms are minimized. In the Dominican Republic it is estimated that more than half of the elderly suffer from this disorder.
\end{abstract}

Objective: Estimate the incidence of depressive illness in elderly adults in Arroyo Hondo, Peravia, Dominican Republic, during the period of November - December 2018.

Methodology: Prospective, descriptive and cross-sectional study. 201 individuals over 65 years were interviewed and the Geriatric Depression Scale (GDS-15) was applied.

\footnotetext{
d Asesor de investigación. Psiquiatra, psicoterapeuta.

e Asesora de investigación. Médico Asistente de Psiquiatría.

${ }^{\mathrm{f}}$ Coordinador de investigación del Instituto Tecnológico de Santo Domingo (INTEC), República Dominicana

g Coordinador de investigación del Instituto Tecnológico de Santo Domingo (INTEC), República Dominicana
}

\footnotetext{
a Egresado del Instituto Tecnológico de Santo Domingo (INTEC), República Dominicana. Autor para
Correo-e: jccastillo.u@gmail.com

b Egresado del Instituto Tecnológico de Santo Domingo (INTEC), República Dominicana. Correo-e: gladisgerdiazv@gmail.com

${ }^{\mathrm{c}}$ Egresado del Instituto Tecnológico de Santo Domingo (INTEC), República Dominicana. Correo-e: jaylenereyes13@gmail.com
} 
Resultados: 168 (84\%) personas no tenían síntomas depresivos; 29 (14\%) se encontraban en el rango de depresión leve y $4(2 \%)$ en el de depresión establecida. Entre las comorbilidades se encontraron HTA, DM, dislipidemia, ECV, entre otras.

Conclusiones: 33 de las personas tienen síntomas depresivos. El pico de mayor incidencia ocurrió entre 75-85 años y, en cuanto al estado civil, los viudos padecieron en mayor frecuencia depresión leve; en los casados se encontraron 2 de los 4 casos de depresión franca. La comorbilidad más frecuente fueron la HTA, seguida de DM y trastornos dislipidémicos.

Palabras clave: depresión; psiquiatría geriátrica; escalas de valoración psiquiátrica; diagnóstico; anciano.

\section{Introducción}

La depresión es un trastorno que difiere de las variaciones habituales del estado de ánimo y de las respuestas emocionales breves a los problemas de la vida cotidiana. Se convierte en un problema de salud serio, especialmente cuando es de larga duración. Puede clasificarse según su intensidad en leve, moderada y grave, y puede causar deserción laboral y problemas escolares y familiares. ${ }^{1}$

Esta entidad es frecuente en los adultos de la tercera edad. La prevalencia de esta afección en la población envejeciente oscila entre el 10-20 \%, según el escenario en que se estudie ${ }^{2}$. Si además se incluyen los individuos con síntomas depresivos que no satisfacen criterios para depresión mayor, la prevalencia aumenta a cifras que llegan hasta el $49 \%$.

A pesar de la elevada prevalencia del trastorno, usualmente es subdiagnosticado y subtratado. El conocimiento y la familiarización del médico y el personal sanitario con dicho trastorno y sus particularidades, son necesarios con el fin de poder realizar la detección y tratamiento oportuno del mismo, y de esta forma reducir el impacto negativo
Results: 168 (84\%) individuals had no depressive symptoms; 29 (14\%) were within the range of mild depression and $4(2 \%)$ had established depression. Among the comorbidities were HTA, DM, dyslipidemia, CVD, and others.

Conclusions: 33 of the people have depressive symptoms. The peak with the highest incidence of depression is found between 75-85 years and, in terms of marital status, widowers suffered most often mild depression, but among those married, 2 of the 4 cases were of established depression. The most frequent comorbidity was HT, followed by DM and dyslipidemic disorders.

Keywords: Depression; geriatric psychiatry; psychiatric evaluation scales; diagnostic; elderly.

que tiene en el paciente, la familia y la comunidad en general ${ }^{3}$.

La presente investigación pretende describir la incidencia de depresión en los adultos de la tercera edad que habitan en la comunidad de Arroyo Hondo, Peravia, durante el período noviembre - diciembre 2018.

Más de un $20 \%$ de las personas que pasan de los 60 años sufren algún trastorno mental o neural (sin contar los que se manifiestan por cefalea) y el 6,6\% de la discapacidad en ese grupo se atribuye a trastornos mentales y neurológicos. Estos trastornos representan en la población anciana un 17,4 \% de los años vividos con discapacidad. Los trastornos mentales y neurológicos más comunes en este grupo de edad son la demencia y la depresión, que a escala mundial afectan aproximadamente al $5 \%$ y al $7 \%$, respectivamente, de la población de adultos mayores ${ }^{4}$. Esto supone un costo de asistencia sanitaria elevado, además de la percepción de "mala salud" por el deterioro que produce ${ }^{4}$.

La enfermedad depresiva en las personas mayores de 65 ańos constituye un problema generalizado y 
desatendido. La etiología es multifactorial; puede ser desencadenada por fenómenos psicosociales, genéticos, de índole biológico a través de mecanismos hormonales, mediados por neurotransmisores y asociado a patologías en las que, a menudo, se minimizan los síntomas afectivos.

Esto no solo incluye afecciones orgánicas, sino aquellas asociadas a la psique que se manifiestan con base en pérdidas características para las personas de la tercera edad: disminución en la capacidad sensorial (visión, audición) y cognitiva (memoria), aunada a la pérdida de seres queridos durante las últimas etapas de la vida, más aún hoy día, donde la esperanza de vida es mucho mayor que antaño.

Otro detalle importante es que las personas mayores con depresión tienen un desempeño más deficiente en comparación con los que padecen enfermedades crónicas como las enfermedades pulmonares, la hipertensión arterial o la diabetes sacarina ${ }^{4}$.

Esta patología, que puede venir sola o acompañada, suele dificultar el desempeño general de la persona que lo padece, disminuyendo su capacidad para afrontar los quehaceres de la vida diaria, e incluso, provocar ideación suicida si llega a estadios avanzados o más complejos 5 .

Debido a que los cambios ocasionados por el envejecimiento aparecen de manera paulatina y varían de un individuo a otro, no existe un consenso que nos permita delimitar los criterios de senectud en todas las sociedades. De hecho, las definiciones de vejez no son consistentes desde el punto de vista de la biología, la demografía, el empleo, la jubilación y la sociología ${ }^{6}$. Sin embargo, para fines estadísticos y administrativos, la vejez se define como la reducción de la competencia para adaptarse a los factores ambientales y se aceptan los 60-65 ańos como el límite inferior de la edad avanzada, aunque la transición personal para convertirse en dependiente ocurre aproximadamente a los 75 ańos ${ }^{7}$.
En ancianos, la depresión también se conoce como depresión tardía o de inicio tardío y se diferencia de la depresión temprana en que esta última tiene un componente familiar depresivo y se asocia a otros trastornos psiquiátricos comórbidos, mientras que en la depresión geriátrica predominan las enfermedades cardiovasculares, disminución de la sustancia blanca, pérdida de las funciones ejecutivas y cambios psicomotores. Ambos trastornos están relacionados con las mismas estructuras corticales y subcorticales y en cuanto a su fisiopatología, si bien ambos trastornos pueden ser diferentes, mismas estructuras neuroanatómicas son responsables de los dos tipos de depresión ${ }^{7}$

Respecto a la edad límite utilizada para definir la depresión geriátrica, existe una falta de consenso. De manera que, algunos de los autores consideran la edad límite de 60 años, mientras que otros la definen como un primer episodio de depresión $\geq 65$ años. $^{8}$

\section{Factores de riesgo}

Para un individuo que ha experimentado períodos de desarrollo saludable, la formación de la integridad del ego y la aceptación de los aspectos negativos y positivos de la vida en su conjunto, la vejez representa un período de maduración e integración de las etapas anteriores. La edad avanzada junto con la amplia experiencia de vida puede proporcionar una ventaja en la lucha contra los factores de estrés. Asimismo, las pérdidas que se producen con la edad, la aparición de limitaciones biológicas y enfermedades físicas, hacen de esta población un grupo de riesgo ${ }^{7}$.

Un meta-análisis de los factores de riesgo de la depresión en los ancianos encontró que el duelo, la alteración del sueño, la discapacidad, la depresión previa y el género femenino son factores de riesgo significativos para la depresión? ${ }^{9}$ En una publicación 
del Centro Nacional para la Información Biotecnológica (NCBI, por sus siglas en inglés), se incluyeron también el vivir solo, divorciarse, bajo nivel de educación, trastorno de funcionalidad, enfermedad física comórbida, disfunción cognitiva de bajo nivel, consumo de cigarrillos y alcohol' .

\section{Pérdida y duelo}

La muerte de un cónyuge se asocia con la disminución de la salud mental y física, el aumento del suicidio y mortalidad por causas distintas a este $\mathrm{y}$ la reducción de ingresos. Durante el primer año de la muerte conyugal, la persona en duelo es propensa a tener más síntomas somáticos, visitas médicas y accidentes. Del 29-58 \% de las personas viudas cumplen con los criterios de depresión mayor al mes y el $25 \%$ todavía los cumple a los tres meses, lo que aumenta notablemente el riesgo de tener una depresión mayor al año. Estudios sugieren que la viudez conduce a tasas más altas de los síntomas depresivos para hombres que para mujeres y que otras pérdidas importantes, como las de hijos y nietos, que pueden ser incluso más devastadoras que la pérdida de un cónyuge ${ }^{10}$.

\section{Enfermedad médica}

Las enfermedades físicas pueden causar síntomas depresivos mediante la limitación de la actividad física de la persona, la reducción de la calidad de vida y la necesidad de dependencia en otros individuos. El $88 \%$ de los adultos mayores tienen una o más enfermedades crónicas y una cuarta parte de este grupo tiene cuatro o más afecciones. Entre el $14 \%$ y el $37 \%$ de los pacientes ambulatorios crónicos tienen síndromes depresivos clínicamente significativos, mientras que, en el caso de los pacientes hospitalizados crónicos, estás cifras alcanzan hasta el $40 \%{ }^{10}$.

En una publicación de la Revista India de Salud Pública (IJPH, por sus siglas en inglés) se determinó que el deterioro funcional era un factor predictor de gran impacto, puesto que los discapacitados funcionales tienen 11 veces más riesgo de depresión que aquellos que sufren de una enfermedad física per se $e^{11}$.

\section{Presentación clínica y diagnóstico}

En atención primaria, los trastornos geriátricos de depresión con frecuencia pasan desapercibidos, de manera que no son detectados a tiempo ni tratados de la manera adecuada. El desafío diagnóstico en este grupo viene dado por los altos índices de enfermedades crónicas, cambios biológicos, problemas de desarrollo socioeconómico relacionados con el envejecimiento, y las presentaciones atípicas de los síntomas. De manera general, las manifestaciones depresivas se atribuyen al envejecimiento normal, al dolor, a la enfermedad física o a la demencia, desaprovechando la oportunidad de iniciar el tratamiento de un padecimiento fácilmente tratable ${ }^{10}$.

Los trastornos del pensamiento más comunes son los delirios de persecución y la idea de considerarse atrapado por una enfermedad intratable. El sentimiento de culpa se ve relativamente menos en este grupo de edad y la existencia de estos sentimientos indica que podría haber habido un episodio depresivo en edades más jóvenes ${ }^{7}$.

Aunque la tendencia de la enfermedad depresiva mayor es a disminuir a medida que se envejece, la incidencia de formas menos severas de depresión clínicamente significativas aumenta de manera constante con la edad y muestra un pico entre mayores de 80 años $^{8,10}$.

La presencia de enfermedades físicas y presentaciones atípicas complican aún más el cuadro clínico. Por lo tanto, se postula que, si bien la presencia de criterios de diagnóstico estándar es necesaria para la depresión en ancianos, esta condición no es sufi- 
ciente para realizar el diagnóstico. Debido a estas complejidades, la depresión en personas de edad avanzada a menudo se clasifica como depresión mayor y no mayor, aquí se incluyen los conceptos de depresión subumbral, depresión subclínica, depresión menor, depresión leve, etc.

\section{Herramientas diagnósticas}

La depresión geriátrica en entornos de atención primaria no se detecta, no se diagnostica y no se trata de manera adecuada ${ }^{10}$. Las evaluaciones breves se pueden administrar a un costo mínimo para el personal y son herramientas disponibles para facilitar la detección de la enfermedad y disminuir los costos generales de atención médica ${ }^{10,12}$.

Una pregunta de selección de un solo elemento es la más simple entre todas las herramientas de evaluación: "¿Se siente a menudo triste o deprimido?" A la que el paciente debe responder, ya sea afirmando o negando. Se probó en una muestra de pacientes con enfermedades médicas en la comunidad y tuvo una sensibilidad del $69 \%$ y una especificidad del $90 \%{ }^{10}$.

Una opción para la detección de la depresión es la Escala de Depresión Geriátrica de 15 ítems (GDS15) es un instrumento desarrollado específicamente para su uso con adultos mayores que se encontró que proporciona resultados confiables y válidos. El GDS-15 tiene un formato de sí-no y está disponible en múltiples idiomas Los puntajes van de 0 a 15 (el aumento de la puntuación se correlaciona con el aumento de la probabilidad de que el individuo esté experimentando depresión). El GDS-15 con una puntuación de corte de 6 tiene una sensibilidad del $94 \%$ y una especificidad del $85 \%$ en adultos que viven en la comunidad, y una puntuación de corte de 5 tiene una sensibilidad del $72 \%$ y una especificidad del $78 \%$ en pacientes de atención domiciliaria $^{12}$.
La Fuerza de Tareas Preventivas de los EE. UU. (USPTF, por sus siglas en inglés) recomienda que todas las personas mayores de 60 ańos sean examinadas periódicamente para detectar la depresión. En términos de un enfoque específico para la detección de la depresión, la USPTF concluyó que, debido a la poca evidencia para recomendar un método de detección sobre otro, los médicos pueden elegir el método que mejor se adapte a sus preferencias personales, a la población de pacientes atendida y al entorno de la práctica. La USPTF también recomienda, la detección de la depresión en adultos siempre que exista un sistema para el diagnóstico, tratamiento y seguimiento ${ }^{12}$.

\section{Pronóstico y tratamiento}

Si bien los adultos mayores tienen menos probabilidades de acceder y recibir servicios de atención de salud mental que sus contrapartes más jóvenes, la depresión tardía se puede tratar tanto con intervenciones psicosociales como con terapias farmacológicas $^{10}$. En este grupo de edad, sin embargo, debido a la existencia de múltiples enfermedades, el uso concomitante de varios medicamentos y la aparición de cambios farmacocinéticos y farmacodinámicos, se requiere de un seguimiento mucho más estrecho cuando se utilizan medicamentos? ${ }^{7}$.

En pacientes ancianos se recomienda iniciar con la dosis mínima de medicamentos psicotrópicos y aumentar de manera progresiva, siempre prefiriéndose el uso de la menor cantidad dentro de lo posible. Se deben utilizar esquemas simples, monitorizar de manera rigurosa los efectos e investigar acerca de las interacciones medicamentosas o enfermedad-medicina antes de iniciar cualquier terapia. ${ }^{7}$

En un meta-análisis y un estudio de revisión sistemática, se determinó que la respuesta a un solo tratamiento farmacológico es baja en la depresión tardía. Sin embargo, la mitad de los pacientes que no 
respondieron al tratamiento sí lo hace al tratamiento adicional o al reemplazo de medicamentos ${ }^{13}$. Se ha probado que la actividad física es útil, y la terapia electroconvulsiva (ECT, por sus siglas en inglés) sigue siendo una opción de tratamiento importante y viable para los adultos mayores con depresión psicótica o severa resistente al tratamiento ${ }^{10}$.

Al comparar las tasas de remisión de ancianos y jóvenes con farmacoterapia y ECT, no se observan grandes variaciones. No obstante, las tasas de recaída son más altas en los adultos mayores ${ }^{7}$, por lo que se aconsejan enfoques de psicoterapia en los que se enfaticen temas como la jubilación, las enfermedades físicas, las disfunciones cognitivas, la separación, el divorcio, la soledad y la muerte ${ }^{7}$.

\section{Material y métodos}

La presente investigación fue de carácter prospectivo, ya que los datos fueron recolectados durante el período noviembre-diciembre 2018, de fuentes primarias; descriptivo, ya que los datos fueron expuestos tal y como se presentaron, y de corte transversal debido a que no se realizó un seguimiento de las variables estudiadas luego de terminado el período de recolección de datos. La población de estudio estuvo conformada por todas las personas mayores de 65 años que pertenecen a la comunidad de Arroyo Hondo, municipio Matanzas, provincia Peravia, República Dominicana, o acudieron a la UNAP de dicha comunidad, durante el período noviembre-diciembre 2018. La población del presente estudio es de 421 personas.

La muestra fue seleccionada a partir del uso de una fórmula estadística para dicho fin, la cual utiliza la población a estudiar, el nivel de confianza, la proporción esperada y la precisión deseada. El resultado fue de 201 personas de una población de 421 para un nivel de confianza de $95 \%$, con una precisión de un $3 \%$ y un $10 \%$ de casos como proporción esperada.
En cuanto a los criterios de inclusión, se determinaron los siguientes: tener más de 65 años; ser residente de la comunidad de Arroyo Hondo o haber asistido a la UNAP "Clínica Rural Arroyo Hondo" y estar de acuerdo en participar en la investigación.

Los datos fueron obtenidos mediante la aplicación, de un formulario (anexo 1), previo consentimiento informado, realizado de manera ambulatoria, casa por casa o en la UNAP, que contenía dos partes: en la primera, se contemplan distintas variables personales, tales como edad, sexo, estado civil, nivel educativo, ocupación, comorbilidades, ingresos económicos del hogar, creencias religiosas y diagnóstico previo de depresión existente; la segunda parte contiene la Escala de Depresión Geriátrica de Yesavage con 15 ítems, con respuestas de sí y no.

Los datos fueron procesados utilizando los programas Microsoft Excel y Microsoft Word, ambos de la suite ofimática de Microsoft "Office 365". Los resultados obtenidos se exponen con gráficos y tablas estadísticas luego de haber sido analizados y clasificados.

\section{Resultados}

Gráfica 1. Incidencia de depresión según la GDS-15 $(\mathrm{n}=201)$

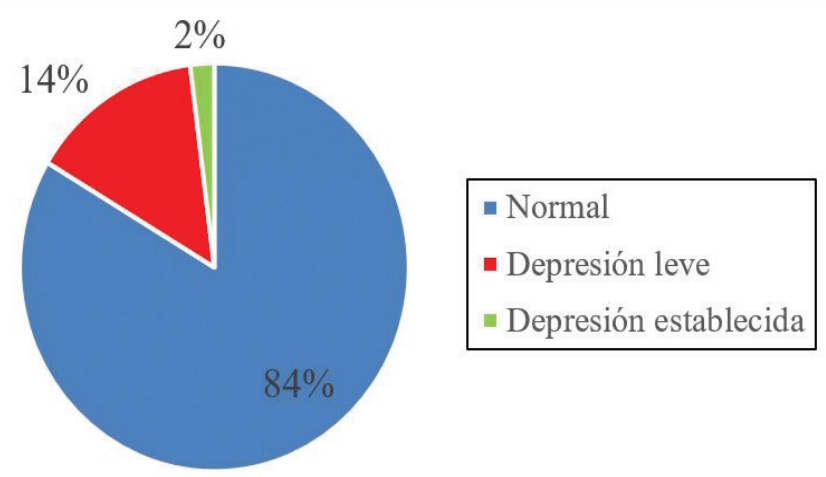

Fuente: elaboración propia. 
La gráfica 1 muestra el porcentaje de personas con depresión. La muestra se clasificó, según la Escala de Depresión Geriátrica de 15 ítems (GDS-15), en normal, a aquellas personas con una puntuación de 0-5; depresión leve, una puntuación de 6-9; y depresión establecida, las personas con una puntuación de 10-15. De una muestra de 201, un total de 168 (84\%) personas obtuvieron valores dentro del rango normal; 29 (14\%) estaban dentro del rango de depresión leve y $4(2 \%)$ presentaron una depresión establecida.

Gráfica 2. Sexo de las personas diagnosticadas con depresión según la GDS-15 (n=201)

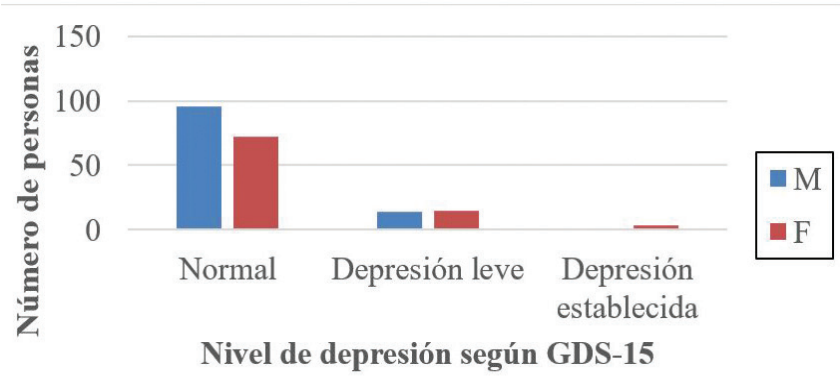

Fuente: elaboración propia.

En la gráfica 2 se puede observar el número de personas, según su sexo, correspondiente a cada nivel de depresión de acuerdo a la GDS-15. De una muestra de 201 personas, 111 (55\%) fueron del sexo masculino. Dentro de los que obtuvieron resultados normales un $57 \%$, correspondiente a 96 personas fueron del género masculino, y el $43 \%$ restante, es decir, 72 personas, del género femenino. Con respecto a la depresión leve, el mayor porcentaje, $52 \%$ (15 personas) fue del género femenino, mientras que $48 \%$ (14 personas) fue del género masculino. Por otra parte, del total de cuatro personas pertenecientes al nivel de depresión establecida, un $75 \%$ (3 personas) fue del sexo femenino, y $25 \%$ (1 persona), del sexo masculino.
Grupos etarios de las personas diagnosticadas con depresión

Para fines estadísticos se clasificaron las personas en tres categorías, vejez joven (personas entre los 65 y 74 ańos), vejez (personas entre 75 y 85 años) y vejez avanzada (personas mayores a 85 años). Un $55 \%$ (110) se encontraban dentro del grupo etario vejez joven, de los cuales el $88.18 \%$ (97) no presentaron indicios de depresión; con depresión leve hubo un total de 11 personas y 2 con depresión establecida, el equivalente a $10 \%$ y $2 \%$, respectivamente. Por otra parte, 72 personas tenían edades entre 75 y 85 años, representando el $36 \%$ de la muestra. De estas 72 personas dentro del rango de vejez, 55 no tenían indicativos de depresión, correspondiente a un $76 \%$; un $21 \%$ de los mismos, equivalente a 15 personas, presentó signos y síntomas de depresión leve; y los 2 restantes, siendo el $3 \%$, sí alcanzaron valores de depresión establecida. Con respecto al grupo de vejez avanzada, este representó un $9 \%$ de la muestra con 19 personas. Dentro de este grupo etario un $84 \%$ o 16 personas, estuvieron dentro del rango de valores normales y el $16 \%$ restante, equivalente a 3 personas, obtuvo valores de depresión leve.

\section{Estado civil de las personas con depresión}

De una muestra de 201 personas, 28 (14\%) personas se encontraban solteras. De las 28 personas solteras, 25 estuvieron dentro del rango normal, dos personas con depresión leve y 1 con depresión establecida, siendo $89 \%, 7 \%$ y $4 \%$, respectivamente. Un total de $65(32 \%)$ personas se encontraban casadas. De las 65 personas casadas, 56 con valores dentro del rango normal, siete con depresión leve y dos con depresión establecida, siendo esto $86 \%, 11 \%$ y $3 \%$ del total de personas casadas. A su vez, otras 65 $(32 \%)$ personas estaban viudas. De las 65 personas viudas, 51 personas no presentaron indicios de depresión y 14 alcanzaron un puntaje de depresión leve, correspondiente con un $78 \%$ y $22 \%$. Por 
otra parte, en unión libre hubo un total de 36 (18\%) personas. Un total de 30 personas de los que se encontraban en unión libre resultaron con valores normales, representando un $83 \%$ de esta categoría; mientras que cinco personas, es decir un $14 \%$, están dentro de depresión leve y un $3 \%$, o sea, una persona, presenta signos y síntomas de depresión mayor. Con respecto a los divorciados, estos representaron un $3 \%$ de la muestra, contando con un total de siete personas. De estas siete personas, $6(86 \%)$ sin depresión, y 1 (14\%) persona con depresión leve.

Tabla 1. Escolaridad y depresión (n=201)

\begin{tabular}{|c|c|c|c|c|}
\hline $\begin{array}{c}\text { Escolari- } \\
\text { dad }\end{array}$ & $\begin{array}{c}\text { Nor- } \\
\text { mal }\end{array}$ & $\begin{array}{c}\text { Depresión } \\
\text { leve }\end{array}$ & $\begin{array}{c}\text { Depresión esta- } \\
\text { blecida }\end{array}$ & $\begin{array}{c}\text { To- } \\
\text { tal }\end{array}$ \\
\hline N/A & 30 & 12 & 2 & 44 \\
\hline $\begin{array}{c}\text { 1ro a 3ro } \\
\text { de básica }\end{array}$ & 76 & 8 & 1 & 85 \\
\hline 4to a 8vo & 52 & 7 & 1 & 60 \\
\hline Bachillerato & 7 & 0 & 0 & 7 \\
\hline Profesional & 3 & 2 & 0 & 5 \\
\hline Total & 168 & 29 & 4 & 201 \\
\hline
\end{tabular}

Fuente: elaboración propia.

La tabla 1 muestra el nivel de escolaridad alcanzado por las personas diagnosticadas con depresión según la GDS-15. Del total de la muestra, el 85.6\% (168), recibió una puntuación menor o igual a 5 , independientemente del nivel de educación hasta el que asistieron. El 14.4 \% (29) cayó en la categoría de depresión leve. Únicamente cuatro individuos reunieron las características clínicas para atribuírseles depresión establecida, de estos, dos no fueron a la escuela; de los restantes 2, uno no logró avanzar hasta 4to de primaria y otro no completó el 8vo curso.
Fuente de ingresos económicos de los pacientes con depresión

De la muestra, 150 personas afirmaron estar recibiendo apoyo económico de terceros, 41 personas aún seguían trabajando y proveyendo para el hogar, y una minoría de 16 personas dependían económicamente de alguna pensión. De las 150 personas que dependían económicamente de otros, 124 (83\%) no presentaron indicios de depresión; mientras que 22 (15\%), sí obtuvieron puntuaciones de depresión leve; y las 4 personas restantes estaban en el rango de depresión establecida. Por otra parte, de las 41 personas que afirmaron ser trabajadores activos, en un $90 \%$ (37), no se observaron indicativos de depresión; mientras que el $10 \%$ (4) restante, presentó síntomas de depresión leve. Dentro de los 16 que refirieron estar recibiendo alguna pensión, 14 personas obtuvieron puntuaciones dentro de los valores normales y dos personas de depresión leve, correspondiente a un $88 \%$ y $12 \%$, respectivamente. Cabe destacar que algunos de los encuestados afirman tener más de una fuente de ingreso.

Tabla 2. Comorbilidades vs. Depresión

\begin{tabular}{|c|c|c|c|c|}
\hline Patología & Normal & $\begin{array}{c}\text { Depre- } \\
\text { sión } \\
\text { leve }\end{array}$ & $\begin{array}{c}\text { Depresión } \\
\text { establecida }\end{array}$ & Total \\
\hline HTA & 106 & 17 & 4 & 127 \\
\hline DM & 28 & 6 & 2 & 36 \\
\hline Dislipidemia & 11 & 3 & 0 & 14 \\
\hline ECV & 10 & 1 & 1 & 12 \\
\hline Osteoporosis & 11 & 1 & 0 & 12 \\
\hline Alzheimer & 3 & 0 & 0 & 3 \\
\hline N/A & 33 & 6 & 0 & 39 \\
\hline
\end{tabular}

Fuente: elaboración propia. 
En la tabla 2 se pretende recoger la información pertinente a la relación entre comorbilidades y la incidencia de depresión. Cabe destacar que en varias instancias los pacientes cargaban con más de una patología a la vez, por lo cual la sumatoria del total de patologías es superior a 201.

\section{Relación entre religión y nivel de depresión}

De una muestra de 201 personas, el $14 \%$ no se siente identificado con ninguna religión en particular, lo que representa un total de 29 personas. De estas 29 personas, el $72 \%$ estuvo dentro del rango de puntuaciones normales, otras 6 personas, es decir, $21 \%$ de esta categoría, presentó signos y síntomas de depresión leve, mientras que el $7 \%$ restante, correspondiente a dos personas, alcanzaron valores de depresión establecida. Por otra parte, el $59 \%$ de la muestra se considera cristiano católico, equivalente a un total de 118 personas. Dentro de estas 118, 102 personas, es decir, $86 \%$, mantuvieron un puntaje por debajo del rango depresivo; mientras que otras 14 , o sea, $12 \%$, alcanzó valores de depresión leve y el restante $2 \%$, correspondiente a dos personas, presentó indicios de una depresión establecida. Un 23 \% de la muestra afirmó ser cristiano evangélico, siendo esto un total de 47 personas, de las cuales 40 , es decir, un $85 \%$ obtuvo valores dentro del rango normal y el otro $15 \%$, equivalente a 7 personas, presentó valores de depresión leve. Un $2 \%$ de los entrevistados se consideró cristiano adventista, lo que corresponde a un total de 4 personas, de los cuales 3 no tenían indicios de depresión y 1 mostró síntomas de depresión leve, $75 \%$ y $25 \%$, respectivamente. Un total de 3 personas pertenece a los Testigos de Jehová, representando un $1 \%$ de la muestra y dentro de estos, 2 , resultando en un $67 \%$ de esta categoría, no tienen indicativos de depresión según la GDS-15, mientras que el $33 \%$ restante, correspondiente a 1 persona, mostró síntomas de depresión leve.

\section{Discusión y conclusiones}

Según los resultados obtenidos en esta investigación, la incidencia de depresión fue del $16 \%$. Estos hallazgos son congruentes con los de la publicación de la South-African Family Practice Journal titulado "Depresión en adultos mayores: prevalencia y factores de riesgo en una muestra de atención primaria de salud", en la que se estipula que entre el 10-20\% de la población general envejeciente tiene depresión ${ }^{14}$. Sin embargo, contrastan con los datos ofrecidos por la OMS en la sección "Salud mental de envejecientes" en la que se estima que la prevalencia es de aproximadamente un $7 \%{ }^{4}$.

Si bien la edad es un factor predictor de importancia en la depresión geriátrica, con una tendencia usual al incremento con la edad ${ }^{15}$, el pico de incidencia resultante en esta investigación ocurrió en aquellas personas entre los 75 y 85 años, para luego disminuir de manera notable al alcanzar la vejez avanzada. Una disposición similar fue expuesta en una investigación titulada "Prevalencia y diferencias de género en la depresión tardía: un estudio basado en la población" divulgado por el American Journal of Geriatric Psychiatry, en la que encontraron que a partir de los 81 ańos la ocurrencia de depresión desciende conforme se avanza en edad. ${ }^{16}$

Independientemente de la edad, ambos sexos se vieron afectados de manera similar por esta enfermedad, aunque en mujeres se observó mayor índice de depresión establecida ${ }^{16}$. Estos resultados se ven respaldados por los obtenidos en una revisión sistemática del Ethiopian Journal of Health Science, en los que se encontró que el sexo masculino es $63 \%$ menos propenso a desarrollar depresión que el sexo femenino ${ }^{17}$.

Con relación al grado académico, se puede observar en los resultados que a menor grado académico alcanzado, mayor es el índice de depresión. Esto se 
corrobora en el informe "Panorama de la Educación 2017” de la Organización para la Cooperación y el Desarrollo Económicos (OCDE) donde se plantean premisas similares con base en diversas investigaciones. Nótese que las 4 personas que tuvieron síntomas francos de depresión clínica ya establecida no culminaron con sus estudios de nivel básico $^{18}$.

En cuanto al estado civil, un $22 \%$ de las personas en estado de viudez quedaron dentro del rango de depresión leve según la GDS-15. En una investigación titulada "Depresión geriátrica en atención primaria", se describió que del 29-58\% de los enviudados cumple con los criterios de depresión mayor durante el primer año ${ }^{12}$. Aunque en los resultados se obtuvo que la mayoría de los afectados eran viudos en cuanto al estado civil se refiere, reafirmando el enunciado anterior, según su puntuación en la GDS-15, este grupo quedó en el rango de depresión leve, no de depresión mayor.

Cuando se trata de la depresión establecida, los resultados de esta investigación fueron contrarios a los esperados, ya que, de los cuatro pacientes diagnosticados, tres tenían su pareja estable y solo 1 se encontraba soltero. En un meta-análisis llamado "Estado civil y riesgo de depresión tardía: un metaanálisis de la literatura publicada", se establecía que, comparado con las personas casadas, los adultos mayores viudos, solteros y divorciados tenían un riesgo mayor de depresión, lo que se opone a los resultados de esta investigación ${ }^{19}$. Sin embargo, en el caso particular de dos de las personas con depresión establecida y que se encontraban casadas, se observó que las parejas respectivas se encontraban discapacitadas y dependían totalmente del cuidado que estas les proporcionaban, lo que añade un factor estresante que las hace susceptibles a sufrir de depresión. Es cierto que no toda persona que cuide de un familiar o ser amado ha de sufrir de depresión, no obstante, según un reporte de la Alianza de Cuida- dores Familiares, del $40 \%$ - $70 \%$ de los cuidadores tienen síntomas de depresión ${ }^{20}$, por lo que los autores de esta investigación proponen que realicen estudios posteriores en este aspecto en específico.

En el aspecto de las creencias religiosas, $85.5 \%$ (172/201) refirió pertenecer judeocristianismo en cualquiera de sus acepciones, a través de una pregunta abierta sobre la religión en el formulario de recolección de datos. Entendemos, debido a razones culturales, que buena parte de los que profesan ser cristianos católicos en realidad no pertenecen a dicha religión y solo repiten su asociación a esta, puesto que "con esa religión fue con la que crecieron", aun cuando sus creencias sean distintas a dicha doctrina.

Esto quizás influye en que de los cuatro individuos con depresión establecida, dos afirman ser cristianos católicos y otros 2 dijeron no pertenecer a ninguna religión. Este hallazgo es importante, debido a que se han relacionado previamente, de manera inversamente proporcional, los síntomas depresivos con el afrontamiento religioso. Esta asociación persiste aun cuando se han esclarecido otros factores sociodemográficos ${ }^{21}$.

La población que se encuentra en la vejez se ha considerado un grupo potencialmente vulnerable y con altas tasas de dependencia económica al no tener recursos suficientes para satisfacer sus necesidades en cantidad y calidad. Como consecuencia de esto, el depender económicamente de otros individuos e incluso de las ayudas proporcionadas por el Estado altera la esfera psicosocial de los individuos con sentimientos de inutilidad y desánimo. Así, diversos estudios señalan que el contar o no con ingresos suficientes en la etapa de la vejez es un predictor para el deterioro de la calidad de vida de las personas mayores, ocasionando que se encuentren expuestos a una mayor prevalencia de trastornos afectivos, cognitivos y conductuales, tales como la 
depresión y la demencia senil ${ }^{22}$. Esta información proporcionada por el artículo "Prevalencia de depresión en hombres y mujeres mayores en México y factores de riesgo", va acorde con los resultados de esta investigación, ya que el mayor número de pacientes con depresión leve se encontraba en las categorías de personas que no se estaban en el momento trabajando o proveyendo para el hogar y el $100 \%$ de los pacientes con depresión establecida pertenecían, específicamente, al grupo de personas que dependían totalmente de la ayuda económica de terceros.

La comorbilidad más frecuente fue hipertensión arterial seguida de diabetes mellitus, con 106 y 28 pacientes, respectivamente. Del total, solo 29 presentaron alguna forma de depresión. Cabe destacar que, de los 4 pacientes con depresión establecida, 2 son manejados por hipertensión, mientras que los otros dos presentan alteración de los niveles tensionales y diabetes. Esto es traído a colación, ya que en revistas como Aging and Disease y The Indian Journal of Psychiatry existen publicaciones en las que se hacen mención de ciertos antihipertensivos como causantes conocidos de depresión ${ }^{7,8}$.

\section{Bibliografía}

1. Organización Mundial de la Salud (OMS). Depresión. [Online]. Available from: https:// www.who.int/es/news-room/fact-sheets/detail/ depression [Accessed noviembre 2018].

2. Barua A, Kumar-Ghosh M, Kar N, Basilio MA. Prevalence of depressive disorders in the elderly. Annals of Saudi Medicine. 2011 Nov-Dec;31(6): 620-4.

3. Peña-Solano DM, Herazo-Dilson MI, Calvo-Gómez JM. Depresión en ancianos. Revista de la Facultad de Medicina. 2009;57(4): 347-55.
4. Organización Mundial de la Salud (OMS). OMS | La salud mental y los adultos mayores. [Online]. Available from: http://origin.who. int/mediacentre/factsheets/fs381/es/ [Accessed diciembre 2018].

5. Organización Mundial de la Salud (OMS). OMS | Depresión. [Online]. Available from: https://www.who.int/topics/depression/es/ [Accessed diciembre 2018].

6. Encyclopaedia Britannica. Old Age| Britannica. com. [Online]. Available from: https://www. britannica.com/science/old-age [Accessed diciembre 2018].

7. Sözeri-Varma G. Depression in the Elderly: Clinical Features and Risk Factors. Aging and Disease. 2012 diciembre;3(6): 465-71.

8. Avasthi A, Grover S. Clinical Practice Guidelines for Management of Depression in Elderly. Indian Journal of Psychiatry. 2018 febrero; 60(3): S341-S362.

9. Cole MG, Dendukuri N. Risk Factors for Depression Among Elderly Community Subjects: A Systematic Review and Meta-Analysis. American Journal of Psychiatry. 2003;160: 1147-56.

10. Park M, Unützer J. Geriatric Depression in Primary Care. Psychiatric Clinics of North America. 2011 Junio;34(2): 469-X.

11. Sengupta P, Benjamin A. Prevalence of depression and associated risk factors among the elderly in urban and rural field practice areas of a tertiary care institution in Ludhiana. Indian Journal of Public Health. 2015;59(1): 3-8.

12. Vieira ER, Brown E, Raue P. Depression in Older Adults: Screening and Referral. Journal of Geryatric Psychiatry. 2014 enero/marzo;37(1): 24-30. 
13. Cole MG, Bellavance F, Mansour A. Prognosis of Depression in Elderly Community and Primary Care Populations: A Systematic Review and Meta-Analysis. American Journal of Psychiatry. 1999 agosto; 156(8): 1182-9

14. Padayachey U, Ramlall S, Chipps J. Depression in older adults: prevalence and risk factors in a primary health care sample. South African Family Practice. 2017;59(2): 61-66

15. Zalavadiya DD, Banerjee A, Sheth AM, Rangoonwala M, Mitra A, Kadri AM. A Comparative Study of Depression and Associated Risk Factors among Elderly Inmates of Old Age Homes and Community of Rajkot: A Gujarati Version of the Geriatric Depression Scale-Short Form (GDS-G). Indian Journal of Community Medicine. 2017 octubre-diciembre;42(4): 204-208

16. Forlani C, Morri M, Ferrari B, Dalmonte E, Menchetti M, De-Ronchi D et al. Prevalence and Gender Differences in Late-Life Depression: A Population-Based Study. The American Journal of Geriatric Psychiatry. 2014 abril;22(4): 370-80.

17. Hassen-Abate K. Gender Disparity in Prevalence of Depression Among Patient Population: A Systematic Review. Ethiopian Journal of Health Science. 2013 noviembre; 23(3): 283-8.

18. Organización para la Cooperación y el Desarrollo Económicos (OCDE). Panorama de la educación 2017. España: Ministerio de Educación, Cultura y Deporte, Subdirección General de Documentación y Publicaciones; 2017.
19. Yan XY, Huang SM, Huang CQ, Wu WH, Qin Y. Marital status and risk for late life depression: a meta-analysis of the published literature. The Journal of International Medical Research. 2011;39(4): 1142-54.

20. WebMD Medical Reference. Why Caregiving Can Lead to Depression. [Online]. Available from: https:/www.webmd.com/healthy-aging/ caregiving-depression\#1 [Accessed diciembre 2018].

21. Koenig HG, Cohen HJ, Blazer DG, Pieper C, Meador KG, Shelp F et al. Religious coping and depression among elderly, hospitalized medically ill men. The American Journal of Psychiatry. 1992 diciembre;149(12): 1693-700.

22. De-Los-Santos PV, Carmona-Valdés SE. Prevalencia de depresión en hombres y mujeres mayores en México y factores de riesgo. Población y Salud en Mesoamérica. 2018 enero-junio; 15(2): $1-22$

23. Dirección General de Desarrollo y Ordenamiento Territorial (DGODT). Plan Municipal de Desarrollo Baní. Baní: Ayuntamiento Municipal de Baní, Consejo de Desarrollo Municipal Baní; 2016.

24. Barias Melo M. Arroyo Hondo, Baní y su Historia. (2nd ed). Peravia, Baní: E\&L; 2009.

25. American Psychiatric Association. Diagnostic and Statistical Manual of Mental Disorders DSM-5. (5th ed). Washington, DC: American Psychiatric Publishing; 2013. 
Incidencia de enfermedad depresiva en adultos de la tercera edad, en Arroyo Hondo, Peravia, República Dominicana, durante el período noviembre-diciembre 2018

\section{Anexos}

Anexo 1. Formulario de recolección de datos

Incidencia de Enfermedad Depresiva en Adultos de la Tercera Edad, en Arroyo Hondo, Peravia, República Dominicana, durante el período noviembre - diciembre 2018.

\section{Datos personales}

\section{Nombre:}

Sexo:
Masculino
Femenino

Edad:

Estado civil:
Soltero/a
Divorciado/a
Casado/a
Unión libre
Viudo/a

\section{Escolaridad:}

\section{Ocupación:}

Fuente de ingresos económicos:

Comorbilidades:

\section{Religión:}

\section{Diagnóstico anterior y/o tratamiento:}


Jean C. Castillo, Gladisder Díaz Vásquez, Jaylene Reyes Montilla, Secundino Palacios, Ysabel Díaz Castro, Emilton López y Leopoldo Reyes Nin

Anexo 2. Formulario de recolección de datos y Escala de Depresión Geriátrica de Yesavage.

\section{Escala de Depresión Geriátrica (EDG)}

Instrucciones: Escoja la mejor respuesta acerca de cómo se ha sentido a lo largo de la última semana.

\begin{tabular}{|c|c|c|}
\hline Pregunta a realizar & Respuesta & Puntaje \\
\hline ¿Está básicamente satisfecho con su vida? & SÍ / NO & \\
\hline ¿Ha renunciado a muchas de sus actividades y pasatiempos? & Sí / NO & \\
\hline ¿Siente que su vida está vacía? & SÍ / NO & \\
\hline ¿Se encuentra a menudo aburrido? & SÍ / NO & \\
\hline ¿Se encuentra alegre y optimista, con buen ánimo casi todo el tiempo? & SÍ / NO & \\
\hline ¿Teme que le vaya a pasar algo malo? & SÍ / NO & \\
\hline ¿Se siente feliz, contento la mayor parte del tiempo? & SÍ / NO & \\
\hline ¿Se siente a menudo desamparado, desvalido, indeciso? & SÍ / NO & \\
\hline ¿Prefiere quedarse en casa que acaso salir y hacer cosas nuevas? & SÍ / NO & \\
\hline ¿Le da la impresión de que tiene más fallos de memoria que los demás? & Sí / NO & \\
\hline ¿Cree que es agradable estar vivo? & Sí / NO & \\
\hline ¿Se le hace duro empezar nuevos proyectos? & SÍ / NO & \\
\hline ¿Se siente lleno de energía? & Sí / NO & \\
\hline ¿Siente que su situación es angustiosa, desesperada? & SÍ / NO & \\
\hline \multirow[t]{2}{*}{ ¿Cree que la mayoría de la gente vive económicamente mejor que usted? } & Sí / NO & \\
\hline & TOTAL & \\
\hline
\end{tabular}

(Sheikh \& Yesavage, 1986)

70 | Ciencia y Salud 2020; 4(1, enero-abril): 57-70 • Casos clínicos 